\title{
Cutaneous cryptococcosis in HIV patient: a diagnostic dilemma
}

\author{
Fatai O. Olanrewaju ${ }^{1}$, Mufutau M. Oripelaye ${ }^{1}$, Olufemi O. Ariyibi $^{2}$ \\ ${ }^{1}$ Department of Dermatology and Venereology, Obafemi Awolowo University, Ile-Ife, Nigeria \\ ${ }^{2}$ Department of Histopathology, Federal Medical Centre, Owo, Nigeria
}

\begin{abstract}
Cutaneous cryptococcosis is an opportunistic infection commonly found in patients with immunosuppression, especially HIV/AIDS, hematological malignancy such as leukemia and lymphoma, diabetes mellitus, sarcoidosis, cancer chemotherapy, and solid organ transplant. The disease has also been occasionally reported in immunocompetent individuals. Although the burden of HIV/AIDS is high in Nigeria, cutaneous cryptococcosis is rarely found. It is caused by ubiquitous encapsulated basidiomycetes yeast-like fungi Cryptococcus neoformans and C. gattii. These two distinct species consist of five serotypes $\mathrm{A}, \mathrm{D}$, and $\mathrm{AD}$ as seen in C. neoformans, and serotype $\mathrm{B}$ and $\mathrm{C}$ are found with C. gattii. The emergence of cutaneous cryptococcosis can provide a window of opportunity for early clinical diagnosis and treatment of more devastating disease such as cryptococcal meningitis with its high mortality of $70-80 \%$. So, physician should be aware of cutaneous cryptococcosis mimicking other cutaneous diseases like Kaposi sarcoma, molluscum contagiosum to help in early diagnosis and treatment. We report a rare case of female HIV patient with cutaneous cryptococcosis of the face, misdiagnosed as Kaposi sarcoma, and adverse drug reaction. At the time of this report, there was no evidence of any systemic disease. She was successfully treated with oral fluconazole monotherapy and to continue life-long oral fluconazole therapy. Patient is to be followed-up with regular physical examinations for any possible recurrence. Physicians should be well informed about many forms of clinical presentations and treatments of cutaneous cryptococcosis to reduce the burden of the disease especially in HIV/AIDS.
\end{abstract}

HIV AIDS Rev 2018; 17, 2: 142-145

DOI: https://doi.org/10.5114/hivar.2018.76377

Key words: cutaneous cryptococcosis, HIV, immunosuppression, fluconazole.

\section{Introduction}

Cutaneous cryptococcosis is caused by ubiquitous encapsulated basidiomycetes yeast-like fungi. The organisms belong to the genus Cryptococcus, and only two among its many species are pathogenic in human: C. neoformans and C. gattii. These two distinct species consist of five serotypes. Serotypes A, D, and AD are seen in C. neoformans, while C. gattii has two serotype B and C. The organisms often caused diseases in immunosuppression, which may pres-

Address for correspondence: Dr. Fatai O. Olanrewaju, Department of Dermatology and Venereology,

Obafemi Awolowo University, 220282 Ile-Ife, Osun State, Nigeria,

phone: +2348035765004, e-mail: docjufat@yahoo.com

ent as disseminated infection such as cryptococcal meningitis with very high mortality ranging between $70-80 \%$ [1]. Occasionally, it causes a disease in immunocompetent individuals. The emergence of cutaneous form of cryptococcosis may signal systemic diseases like cryptococcal meningitis or reactivation of pulmonary cryptococcosis. The mortality from systemic cryptococcal infections has been reported to be higher than tuberculosis in sub-Saharan Africa, especially with emergence of HIV co-infection [2] despite Mycobacterium tuberculosis being the commonest opportunistic

Article history:

Received: 20.04.2017

Received in revised form: 07.11.2017

Accepted: 11.01.2018

Available online: 21.05.2018
International Journal of HIV-Related Problem

HIV \& AIDS

R e v i e w 
infection among HIV population. This opportunistic yeast has a worldwide distribution. Cryptococcus neoformans are found mainly in Europe and America associated with avian excreta, particularly pigeons, while the C. gattii are distributed in the tropics and sub-tropics associated mostly with eucalyptus trees and many decaying woods $[3,4]$

\section{Case report}

A 39-year-old Nigeria woman was diagnosed HIV positive in July 2016, when she presented at the general outpatient department of our hospital with two months history of progressive weight loss and multiple crusted ulcers and nodules on the face (Fig. 1). The lesions started as three small papules on the face, and gradually increased in number and size to nodules. Two weeks later, the lesions became ulcerated, crusted, and cosmetically unacceptable to the patient prompting referral to the dermatologist. At time of referral, she was severely immunosuppressed with CD4+ count of 21 cells/ $\mu$ l. She has also been commenced on highly active antiretroviral therapy (HAART) - efavirenz $600 \mathrm{mg} /$ tenovovir $300 \mathrm{mg} / \mathrm{lamivudine} 300 \mathrm{mg}$ - by the referring physician a month earlier. She had earlier consulted other general practitioners (GP) and family physicians with different diagnoses of Kaposi sarcoma and cutaneous drug reaction to oral over-the-counter (OTC) medications like cotrimoxazole and others she could not remember. Based on the diagnosis of drug reaction, she was advised by her GP to stop all her OTC medications except the antiretroviral drugs she started a month earlier. Despite the advice, the papules, nodules, plaques, and ulcers persisted and increased in size. History of fever, cough, chest pains, headache, neck pains and stiffness, and altered level of consciousness were all absent. There was no preceding history of trauma to the face or recent travels. No contact with birds, bird droppings, or handling of woods. History of drug allergy and ingestion of any routine drugs like steroids were negative. She is neither a known diabetes mellitus patient nor asthmatic.

Examination of the patient revealed few umbilicated papules, firm nodules and multiple ulcerated plaques with crusts on the face (Fig. 1). Central nervous system and chest examination were normal findings. She was diagnosed as a case of cutaneous cryptococcosis. Investigations included complete blood count, liver function test, renal function test, fasting blood glucose, lipid profile, and chest X-ray were all within normal limit.

Histopathological findings of the patient were consistent with inflammatory pseudotumor as shown in Figure 4 . These included areas of ulcerations and infiltration of dermis by numerous lymphocytes, plasma cells, and giant cells. Some scattered polygonal and spindle shaped cells were present in the background. No definitive granuloma was seen, although some thin walled vascular channels were present. Cryptococcal inflammatory pseudotumor had been reported in HIV patients, and was considered as the likely cause of this patient condition [5]. The diagnosis may be further confirmed by positive skin biopsy culture for cryptococcal organisms, which appear milky-white and shiny on Sabouraud dextrose agar.

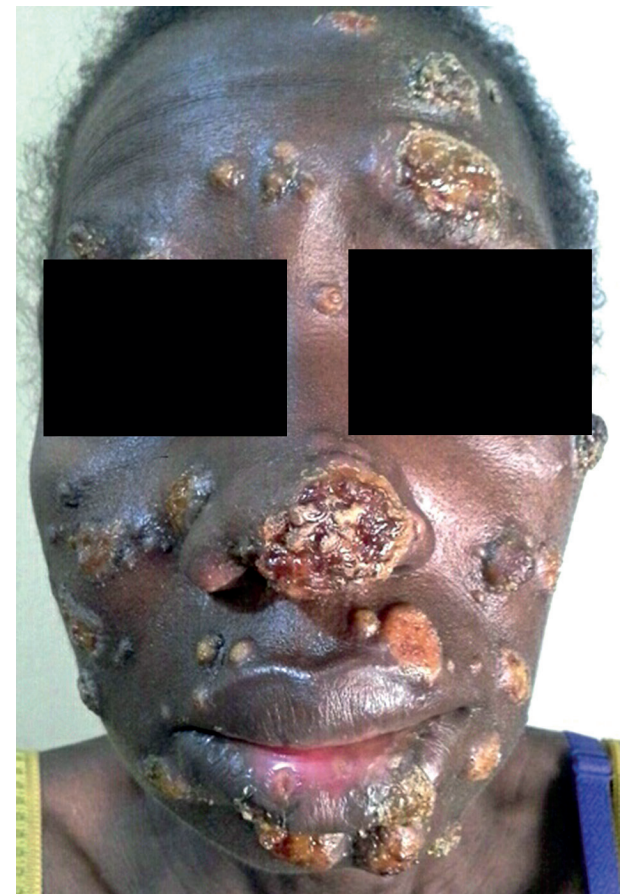

Fig. 1. Crusted ulcers, umbilicated papules, plaques, nodules on the face

The patient was commenced on oral fluconazole monotherapy at a dose of $400 \mathrm{mg} /$ day, while she continued with the highly active antiretroviral therapy. She showed significant improvement one month after the commencement of oral fluconazole as evidenced by rapidly healing lesions (Figs. 2 and 3). In view of the immunosuppression, she was counseled to continue life-long oral fluconazole at reduced dose of $200 \mathrm{mg} /$ day and HAART. The clinical appearance of the lesions especially the umbilicated papules at the nasal bridge and upper lip, histopathology of the skin biopsy, which support cutaneous cryptococcosis but ruled out Kaposi sarcoma (Fig. 4), and response to oral fluconazole supported our initial clinical diagnosis.

\section{Discussion}

Cryptococcal infection was first described by Otto Busse and Abraham Buschke, when they independently isolated the organism from a 31-year-old woman with osteomyelitis of the tibia bone. Both of them presented their findings in a conference at Greifswald Medical Society, Germany in $1894[6,7]$. Their discovery of human cryptococcosis gave rise to the early eponym of Busse-Buschke disease [8]. In the same year, Sanfelice named a similar fungus that was isolated from peach juice Saccharomyces neoformans. Cryptococcus neoformans was finally coined by Jean-Paul Vuillemin for the same yeast in 1901. Since then, more than 70 species have been named by different researchers, of which C. neoformans and C. gattii are pathogenic to human. 


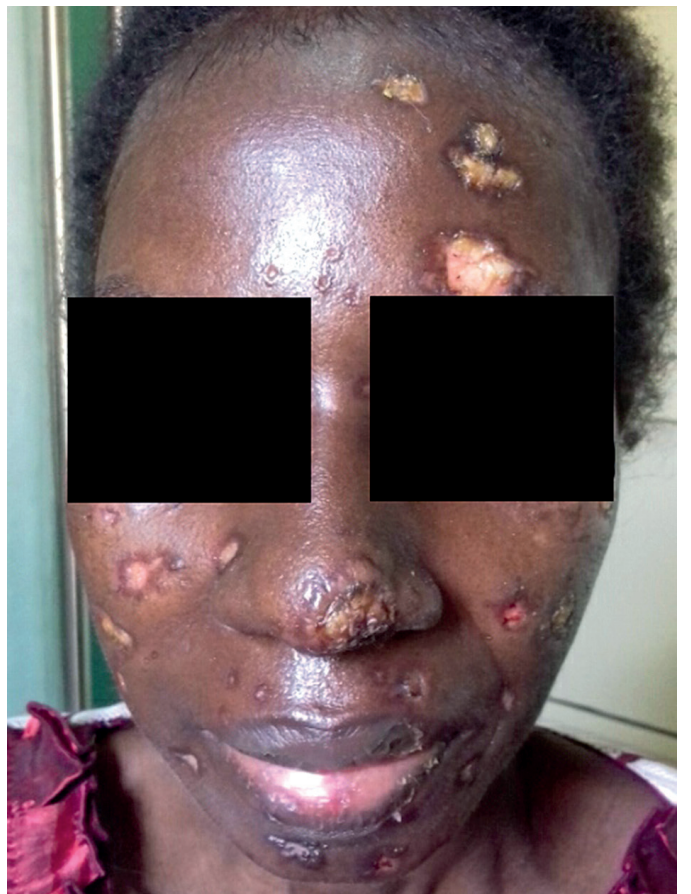

Fig. 2. Healing lesions after one month of fluconazole monotherapy

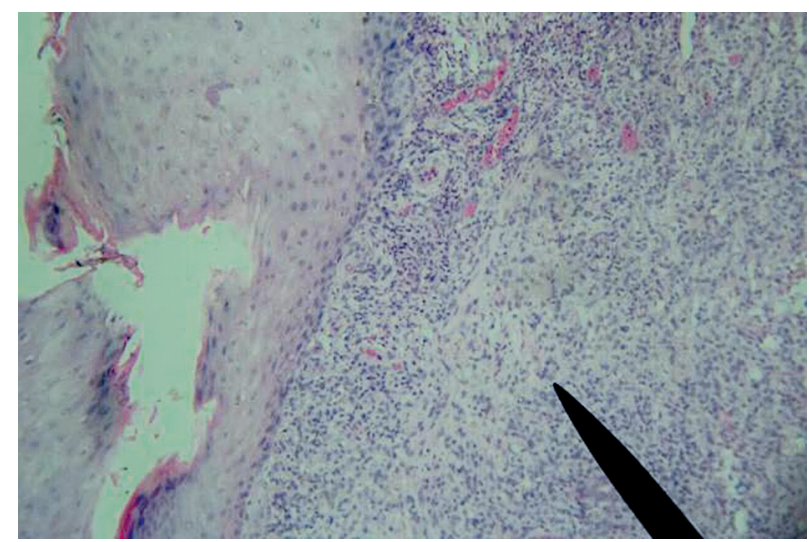

Fig. 4. Histology of skin biopsy section showed ulceration, mixed inflammatory, and multinucleated giant cells (arrowed). Mag. $40 \times 10$

Although cutaneous cryptococcal infection has been described in phenotypically normal healthy population $[9,10]$, it is mostly seen in immunocompromised individuals like HIV/AIDS, as seen in our case report (Fig. 1), prolonged corticosteroid use, hematological malignancy such as leukemia and lymphomas, connective tissue disease like SLE, solid organ transplant on immunosuppressive therapy, cancer chemotherapy, diabetes mellitus, and sarcoidosis [11].

The cutaneous manifestations of cryptococcosis are protean and can resemble other cutaneous diseases like molluscum contagiosum, Kaposi sarcoma, pyoderma gangrenosum,

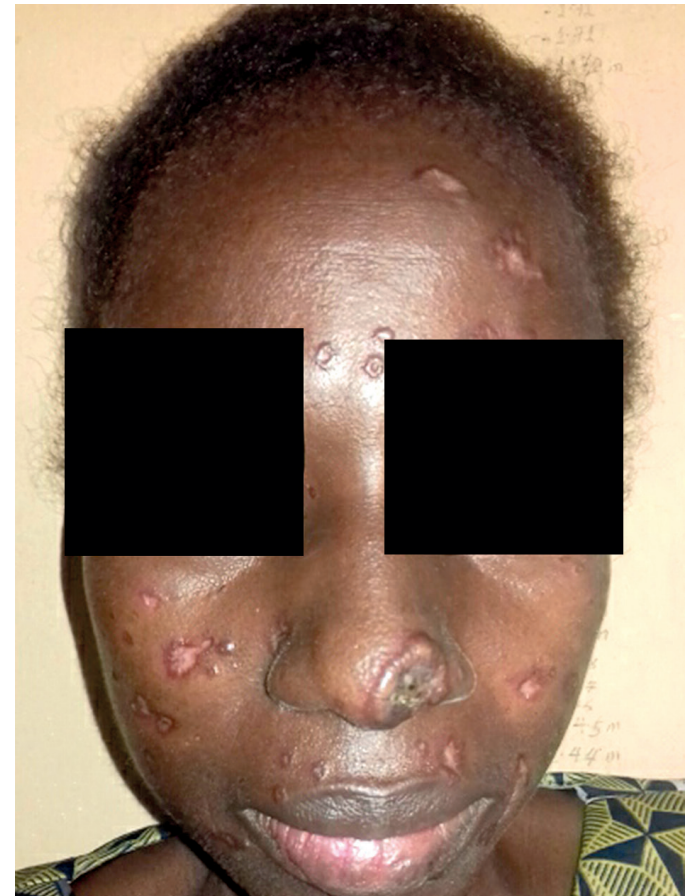

Fig. 3. Healed lesions after four months of fluconazole monotherapy

and basal cell carcinoma (Fig. 1). Other forms of presentation include acneiform, nodular, cellulitis, plaque, papular, ulcerative, crusted [12]. So high index of suspicion and the experience of the attending dermatologist is needed to diagnose the cutaneous form of the disease, especially in resource poor setting with inadequate laboratory facilities like ours.

Cryptococci are able to propagate infection because of the unique structures that conferred the yeast with virulence advantages. The presence of these virulence factors allow the yeast to evade the immune response of the host. Most prominent virulence factor that has been subject of intense research is the polysaccharide capsule, which inhibits phagocytosis [12]. The organism also has the potential ability to survive well in the host environment at $37^{\circ} \mathrm{C}$ due to presence of calcineurin and protease kinase unlike other nonpathogenic cryptococcal species $[13,14]$. Cryptococcus neoformans and C. gattii have ability to synthesis melanin and mannitol, which help the yeasts to withstand oxidative stress from antimicrobial agents and evade phagocytosis $[15,16]$. Other less studied cryptococcal virulence factors are production of proteases, urease, phospholipases, and superoxide dismutase [14].

The presentation of cryptococcosis depends largely on the host cell mediated immunity, size of the inoculum, and the cryptococcal virulence factors. The lungs are the commonest organ to be infected through inhalation of air-borne yeast [17]. In immunocompetent individuals, majority of the host developed a primary focus in the lungs without any signs and symptoms of the disease for a long time. However, in patients with depressed cellular immunity such as HIV, there could be a reactivation of this primary focus, and subsequent second- 
ary hematogenous dissemination to other sites with central nervous system and skin being the commonest organ of involvement [18]. Patients could also present with primary cutaneous cryptococcosis (PCC), which is defined by the presence of cutaneous lesions restricted to a region of the body with no evidence of dissemination to other organs, and the skin culture yielding positive result [19]. Controversies still trail the existence of the primary form of the disease. Some authors believe PCC results from direct inoculation to the skin, while others believe cutaneous cryptococcosis is secondary to hematogenous dissemination [18]. In both forms of the disease, a thorough search for evidence of systemic dissemination and early treatment should be undertaken to prevent a potentially fatal disease like cryptococcal meningoencephalitis. The treatment options depend on the patient's immunity and anatomical sites involved in the disease process. A clinical practice guideline developed by the Infectious Disease Society of America recommends a life-long maintenance therapy using azole for non-disseminated cryptococcosis outside the central nervous system in HIV patients [20]. The drawback of this long-term systemic azole therapy is prolongation of $\mathrm{QT}_{\mathrm{C}}$ interval, potential hepatotoxicity, and drug-drug interactions. Other medications that are more effective include intravenous amphotericin B in combination with oral flucytocin; however, the numerous side-effect of these medications may preclude their regular usage.

\section{Conclusions}

The dermatologist and non-dermatologist, especially those involved in the management of HIV, should be aware of the diagnostic challenges posed by cutaneous cryptococcosis due to its numerous forms of presentations and clinical similarity to other diseases. Though cutaneous cryptococcosis is rarely reported in Nigeria, clinician should have high index of suspicion for this disease during routine medical examination in HIV patients. This will assist significantly in making early diagnosis since cutaneous cryptococcosis can be a pointer to more devastating disseminated infections. Early diagnosis will provide a window of opportunity for early treatment to reduce high morbidity and mortality rate seen with systemic cryptococcosis such as meningoencephalitis, especially in immunosuppression as observed in this case report.

\section{Conflict of interest}

The authors declare no potential conflicts of interest with respect to the research, authorship, and/or publication of this article.

\section{References}

1. Sanchez P, Bosch RJ, de Galvez MV, et al. Cutaneous cryptococcosis in two patients with acquired immunodeficiency syndrome. Int J STD AIDS 2000; 11: 477-480.

2. Park BJ, Wannemuehler KA, Marston BJ, et al. Estimation of the current global burden of cryptococcal meningitis among persons living with HIV/AIDS. AIDS 2009; 23: 525-530.
3. Neuville S, Dromer F, Morin O, et al. Primary cutaneous cryptococcosis: a distinct clinical entity. Clin Infect Dis 2003; 36: 337-347.

4. Ellis DH, Pfeiffer TJ. Natural habitat of Cryptococcus neoformans var. gattii. J Clin Microbiol 1990; 28: 1642-1644.

5. Sing Y, Ramdial PK. Cryptococcal inflammatory pseudotumours. Am J Surg Pathol 2007; 31: 1521-1527.

6. Freij JB, Freij BJ. The Earliest Account of Human Cryptococcosis (Busse-Buschke Disease) in a Woman with Chronic Osteomyelitis of the Tibia. Pediatr Infect Dis J 2015; 34: 1278.

7. Busse O. Ueber parasitare zelleninschlusse und ihre zuchtung. Zentralbl Bakterial 1894; 16: 175-180.

8. Buschke A. Ueber eine durch Coccidien Hervergerufene Krankheit des menschen. Deutsche Med Wochenschr 1895; 2: 14.

9. Leão CA, Ferreira-Paim K, Andrade-Silva L, et al. Primary cutaneous cryptococcosis caused by Cryptococcus Gattii in an immunocompetent host. Med Mycol 2011; 49: 352-355.

10. Pau M, Lallai C, Aste N, et al. Primary cutaneous cryptococcosis in an immunocompetent host. Mycoses 2010; 53: 256-258.

11. John CC, William E, David A. Primary cutaneous cryptococcosis in immunocompetent and immunocompromised hosts. Med Mycol 2003; 41: 177-188.

12. Doering TL. How sweet it is! Cell wall biogenesis and polysaccharide capsule formation in Cryptococcus neoformans. Annu Rev Microbiol 2009; 63: 223-247.

13. Fonseca A, Boekhout T, Fell JW. Cryptococcus Vuillemin (1901). In: The Yeasts: a Taxonomic Sudy. Vol. 3. CP Kurtzman, JW Fell, T Boekhout (eds.). Elsevier, Amsterdam 2011; pp. 1661-1745.

14. Srikanka D, Santiago-Tirado FH, Doering TL. Cryptococcal neoformans: Historical curiosity to modern pathogen. Yeast 2014; 31: 47-60.

15. Alp S. Melanin and its role on the virulence of Cryptococcus neoformans. Mikrobiyol Bul 2010; 44: 519-526.

16. Wong B, Perfect JR, Beggs S, Wright KA. Production of the hexitol D-mannitol by Cryptococcus neoformans in-vitro and in rabbits with experimental meningitis. Infect Immun 1990; 58: 1664-1670.

17. Chayakulkeeree M, Perfect JR. Cryptococcosis. Infect Dis Clin North Am 2006; 20: 507-544.

18. Garcia-Hermoso D, Janbon G, Dromer F. Epidemiological evidence for dormant Cryptococcus neoformans infection. J Clin Microbiol 1999; 37: 3204-3209.

19. Neuville S, Dromer F, Morin O, et al.; French Cryptococcosis Study Group. Primary Cutaneous Cryptococcosis: A Distinct Clinical Entity. Clin Infect Dis 2003; 36: 337-347.

20. Perfect JR, Dismukes WE, Dromer F, et al. Clinical practice guidelines for the management of cryptococcal disease: 2010 update by the infectious diseases society of America. Clin Infect Dis 2010; 50: 291-322. 